\title{
Nonlinear deformations of piezoelectric composite beams
}

\author{
Vahid Tajeddini and Anastasia Muliana ${ }^{*}$ \\ Department of Mechanical Engineering, Texas A\&M University \\ *Corresponding author: amuliana@tamu.edu; phone: 979-458-3579
}

\begin{abstract}
This study presents large deformations of slender elastic and viscoelastic beams with multiple piezoelectric patches attached on their top and bottom surfaces. The slender beams can undergo large in-plane 2-D deformations due to electric fields applied through the piezoelectric patches and mechanical actuations. A nonlinear electro-mechanical constitutive equation is considered for the piezoelectric patches, while linear elastic and viscoelastic constitutive equations are used for the beams. Reissner's finite-deformation beam theory is adopted in formulating the large 2-D deformation, and modified in order to incorporate the deformation due to the electric field input. For an elastic beam, closed form solutions are obtained for the deformations of the beam under electric field actuation, while a nonlinear shooting method is used to analyze the deformation of the beam under both electrical- and mechanical stimuli. For viscoelastic beams, time-dependent deformations of the beams under electric field actuations through the piezoelectric patches are solved numerically. By applying electric fields with different amplitude at different locations of patches, desired deformed shapes in active flexible beams can be achieved, which is useful for analyses and designs of active foldable systems. This study also highlights the effect of viscoelastic materials on the shape changes in foldable electro-active beams.
\end{abstract}




\section{Introduction}

Piezoelectric materials, which are considered as smart materials, experience deformations under applications of electric fields and produce electric charges when subjected to mechanical loads. These materials are commonly used as sensors and actuators in practical applications. As sensors, they can be used to monitor changes in deformations or strains due to pressures or chemical reactions, such as in oil wells. As actuators, they can be used to control deformations or attain shape changes in active structures by applying electric fields through the piezoelectric materials that are integrated to the structures. There have been several studies on analyzing deformations in elastic beams and plates having distributed piezoelectric patches, which are used in vibration controls, acoustic applications, and noise control systems [1]. For example, by using piezoelectric actuators, dynamic performance of aerospace structures are controlled through increasing damping of the structure and decreasing vibration amplitudes [2]. Most of these studies only consider small deformations in the active structures and relatively small amplitude of electric field is needed for vibration suppression.

Recent development in adaptive foldable (flexible) structures allows for controllable reconfiguration into various shapes, which have many engineering applications, e.g. artificial skins, morphing aircraft, flexible robots for use in hazardous environments, etc. One example of achieving active flexible systems is by integrating piezoelectric ceramics into homogeneous polymeric matrix, termed as electro-active composites, which form lightweight flexible (compliant) active materials. When subjected to external stimuli, flexible/compliant systems generally experience large deformations. There have been analytical and numerical studies presented on simulating large deformations of flexible structures, such as slender beams, subjected to a mechanical stimulus. Most of these studies are available for elastic and homogeneous structures. Commonly used analytical methods for obtaining solutions to large 
deformations of elastic beams include power series, equivalent systems [3], e.g., pseudo-linear systems, which solve the nonlinear problems by transforming the equations into a set of linear equations, and elliptical integrals [4,5], while the numerical approaches include Rung-Kutta, shooting method and finite element analyses, see Tada and Lee [6], Yang [7] and Chajes [8]. In the deformation analysis of slender beams undergoing large deformations, the slope of the deflected middle axis cannot be neglected in determining the curvature of the beam. Reissner [9] formulated the governing equations for large 2-D displacements and finite strains of elastic beams subjected to mechanical loadings. Later, Irschik and Gerstmayr [10] derived the Reissner's governing equations for originally straight beams based on continuum mechanics. The governing equations are expressed in the Lagrangian configuration and results in a system of nonlinear differential equations, which can be solved numerically or analytically depending on the prescribed boundary conditions. It is noted that for slender bodies whose dimensions are not of comparable orders of magnitude the bodies can undergo large deformations, mainly to due to large rotations, while the strains in the bodies remain small [11, 12].

There have been limited studies on analyzing nonlinear deformations of smart structures. Lagoudas and co-authors [13-15] studied the deformations of flexible rods with embedded shape memory alloys by using shear-lag model in order to approximate the axial forces and moments induced by the actuators. Banerjee et al. [16] presented nonlinear shooting and Adomain decomposition methods in order to obtain solutions to large deformations of cantilever beams under mechanical loadings, which can be extended to predict large deformations in cantilever beams with piezoelectric materials. A linear elastic constitutive model was considered for the cantilever beams. In actuation applications, it is often necessary to apply high electric field inputs to the piezoelectric components in order to obtain large deformations. When subjected to high 
electric fields, the piezoelectric materials often experience nonlinear electro-mechanical responses, see Tiersten [17]. Tiersten formulated an electro-mechanical constitutive model by considering higher order terms of the electric field in order to describe the nonlinear electromechanical coupling behavior of piezoelectric ceramics (PZT-G) materials. A limited number of studies have considered nonlinear electro-mechanical response of piezoelectric materials due to large electric fields [18-20], but for small deformations. In the present study, higher order terms of the electric field are taken into account in the electro-mechanical constitutive relation of smart beams undergoing large deformations.

In many flexible structures, polymers are widely used because of their capability in undergoing large deformations. One of the prominent characteristics of polymers is their timedependent (viscoelastic) behavior. It might be necessary to consider the time-dependent behaviors in the viscoelastic polymeric structures when non-mechanical stimuli are prescribed in order to obtain shape changes. There are limited studies that address large deformations of viscoelastic beams, e.g., Ya-Peng and Ya-Fei [21], Holden [22], Baranenko [23], Lee [24], Vaz and Caire [25]. Both analytical and numerical solutions of large deformation beams with linear viscoelastic constitutive model have been considered. To the best of our knowledge, very limited studies address nonlinear deformation of active viscoelastic structures. Beldica and Hilton [26] studied stress, deformation and failure of a fiber composite beam with piezoelectric layers by considering general nonlinear anisotropic viscoelastic constitutive relations for piezoelectric layers and the beam. For incorporating large deformations they took into account a large rotation in expressing the curvature of the middle axis of the beam. Muliana [27] presented large deformation analyses of viscoelastic polymeric beams by adopting the Reissner finite strain beam theory. Linear and nonlinear viscoelastic constitutive models are considered, and under 
relatively large external stimuli the linear and nonlinear constitutive models result in significantly different responses.

The present study analyzes nonlinear deformations of active beams having multiple piezoelectric patches, actuated by electric fields. Linear and nonlinear electro-mechanical constitutive models are incorporated for the piezoelectric patches, while elastic and viscoelastic behaviors are considered for the host beams. The first part presents an analysis of large deformations of a smart elastic slender cantilever beam, i.e., homogeneous elastic beam with arbitrary number of piezoelectric actuator patches. The beam is assumed relatively slender so that the effect of the transverse shear deformations on the lateral deflections of the beam can be neglected. The actuators are placed in the form of pairs of piezoelectric patches, which are attached to the top and bottom surfaces of the beam. To induce large deformations and sharp curvatures in the beam, the top and bottom actuators are subjected to large electric fields so that they experience opposite elongation and contraction along the longitudinal axis of the beam. Under such electric field inputs, the beam experiences bending. The governing equations for large deformations in an elastic beam formulated by Reissner [9] are adopted and modified to include the electro-mechanical coupling effect from the piezoelectric patches. It is assumed that the plane that is perpendicular to the longitudinal axis of the undeformed beam remains plane during the deformations, which is a sensible assumption for a slender beam. Analytical solutions of the governing equations are then presented for the deformations of the beams. When the cantilever smart beam is also subjected to mechanical loads, the nonlinear shooting method [16] is used to convert the boundary value problem from the governing equations of the deformations of the beams to an initial value problem, and a $4^{\text {th }}$-Rung-Kutta method is used to numerically solve the initial value problem. The second part of this study considers deformations of smart 
viscoelastic thin beams actuated by electric fields. The piezoelectric patches induce bending moments in the homogeneous beam, which is modeled with linear viscoelastic constitutive relations and the nonlinear Reissner kinematic relations are then considered to obtain the timedependent deformations of the beam.

The outline of the paper is as follows: Section 2 presents the governing equations for large deformations of 2-D elastic beams subject to mechanical and electrical stimuli, followed by a nonlinear shooting method for solving boundary value problems. Section 3 discusses timedependent analyses of viscoelastic beams with piezoelectric patches. Section 4 presents several boundary values problems for both elastic and viscoelastic beams with piezoelectric patches. The present study can be used for preliminary design of active compliant systems. Several parameters, such as number and location of piezoelectric patches and magnitude of electric fields are varied in order to control the deformations of the beam and obtain desired shapes. Section 5 summarizes the present work.

\section{Governing Equations for Active Elastic Beams}

Consider an originally straight cantilever beam under electric field inputs applied to the pair of piezoelectric patches, as shown in figure 1. In order to induce bending in the beam, the electric fields on the top and bottom patches should be applied such that one would cause elongation along the longitudinal axis of the beam while the other would cause contraction. In many practical applications, thin piezoelectric patches are considered and the electric fields are applied through the thickness of the patches. If the top and bottom piezoelectric patches have the same through thickness poling direction, then bending can be obtained by applying electric fields in the opposite directions to the top and bottom patches. 
Crawley and de Luis [28] showed that when the actuator is perfectly bonded to the surface of its host structure, shear force is transferred from the excited actuator to the host structure near the edges of the patch. It is assumed that the piezoelectric patch has significantly smaller length compared to the length of the beam and also it is sufficiently thin so that the stress components in the thickness direction are neglected and the distribution of the extensional strain through the thickness of the actuator is assumed uniform. The transverse surfaces of the beam is assumed to remain straight during the deformation. Moreover, it is assumed that the center line of the beam is inextensible; and in such case large deformation in a slender beam is due to large rotation gradient, while the strain remains small [11, 12]. Therefore, when a small strain measured in considered and the transverse surfaces of the beam is assumed to remain straight during the deformation, the axial strain distribution through the thickness of the host structure due to electric field inputs is linear. The effective bending moment calculated by Wang and Rogers [1] is adopted to determine the bending moment, $M_{0}$, induced by the attached pairs of actuators. Consider a segment of the beam with its middle axis in $\xi$-direction and with a pair of piezoelectric patches bonded on the top and bottom surfaces, as seen in figure 2. The thickness of the bonding agent and its effect on the overall deformations of the beams are neglected. The actuators with the same through thickness poling direction are excited by electric voltages in the opposite directions. The magnitude and direction of the applied electric field to the patches are such that the excited patches induce bending moment to the beam. Incorporating the interaction of the patches and the beam, the bottom actuator undergoes strain $\varepsilon_{a c}$ while the top one undergoes strain $-\varepsilon_{a c}$. The strain in the beam $\varepsilon_{b}$ is assumed linearly distributed through the thickness of the beam, as shown in figure 2 . 
The piezoelectric material is assumed linear elastic with regard it its mechanical behavior, while linear and nonlinear responses are considered for the electro-mechanical coupling. The stress in the excited bottom actuator is expressed as

$$
\sigma_{a c}=E_{p}\left(\varepsilon_{p}-\varepsilon_{a c}\right)
$$

where $E_{p}$ is elastic modulus of the piezoelectric actuator, $\varepsilon_{p}$ is the piezoelectric free strain, which is a strain in the actuator due to an applied electric field without constraints to the actuator. The strain $\varepsilon_{a c}$ is generally smaller than $\varepsilon_{p}$ due to the bonding constraint between the patch and the beam. The strain $\varepsilon_{a c}$ is determined by imposing the compatibility condition between the beam and actuator, and equilibrium equations in terms of axial force and bending moment. The stress in the top patch has the same magnitude as the stress in the bottom patch, shown in Eq. (1), but in compression. Thus, the moment $M_{a c}$ induced by the stressed actuators in Eq. (1) with the respect to middle axis of the beam is expressed as

$$
M_{a c}=t_{p} b\left(t_{p}+t_{b}\right) E_{p}\left(\varepsilon_{p}-\varepsilon_{a c}\right)
$$

where $b$ is width of the beam; $t_{p}$ and $t_{b}$ are the thicknesses of patches and the beam, respectively. If a linear elastic beam is considered, the stress in the beam is given as:

$$
\sigma_{b}=E_{b} \varepsilon_{b}
$$

where $E_{b}$ is the elastic modulus of the beam. Hence, an equivalent bending moment in the beam is obtained from following expression:

$$
M_{0}=-\int_{-\frac{t_{b}}{2}}^{\frac{t_{b}}{2}} E_{b} \varepsilon_{b} \eta b d \eta
$$

Considering the beam segment and the patches in figure 2 as a system, and in absence of any external load; the equilibrium of bending moments about the middle axis of the beam leads to 
$M_{a c}=M_{0}$. By equating Eqs. (2) and (4), the induced moment due to an application of electric

fields is obtained as:

$$
M_{0}=\frac{-b t_{p} t_{b}^{3}\left(t_{p}+t_{b}\right) E_{p} E_{b}}{t_{b}^{3} E_{b}+6 t_{p}\left(\frac{t_{p}}{2}+t_{b}\right)\left(t_{p}+t_{b}\right) E_{p}} \varepsilon_{p}
$$

Assuming that $t_{p}<<t_{b}$, Eq. (6) can be reduced to:

$$
M_{0} \approx \frac{-b t_{p} t_{b}^{2} E_{p} E_{b}}{t_{b} E_{b}+6 t_{p} E_{p}} \varepsilon_{p}
$$

Depending on the magnitude of electric field applied and the response of piezoelectric material, the strain in the piezoelectric patches is expressed as follows:

$$
\varepsilon_{p}=d_{12} E^{e}
$$

when a linear electro-mechanical constitutive relation is considered, which is valid only for a relatively small electric field input. In the above equation, $d_{12}$ is the linear piezoelectric constant, and $E^{e}$ is electric field in thickness direction, $\eta$, of the patch. On the other hand, when a relatively large electric field input is considered, constitutive models with higher order electric fields are used, such as a second order constitutive relation for large electric field [17]:

$$
\varepsilon_{p}=d_{12} E^{e}+\frac{1}{2} \beta_{12} E^{e 2}+H O T
$$

where, $\beta_{12}$ is the quadratic piezoelectric constant and HOT stands for higher order terms.

As mentioned, it is assumed that the top and bottom piezoelectric patches have the same through the thickness poling direction. Also, as discussed, to induce bending moment by a pair of patches on the top and bottom of the beam without an axial elongation in the middle axis (inextensible of the center line) of the beam, $\varepsilon_{p}$ of the top patch should have the same magnitude 
as the one of the bottom patch but with opposite sign. Consequently, according to Eq, (8), if the piezoelectric material show a linear behavior with respect to electric field, the magnitude of the electric field applied to the top patch should be the same as the one to the bottom patch but in opposite direction, e.g.

$$
\left.E^{e}\right|_{\text {top }}=-\left.E^{e}\right|_{\text {bottom }}
$$

where the negative sign stands for the opposite direction. For a nonlinear behavior of the actuators according to Eq. (9), the applied electric field in the top patch with respect to the one in the bottom patch has different magnitude and may be in same direction or opposite direction as expressed in Eq. (11) and Eq. (12), respectively, while keeping the center line inextensible.

$$
\begin{aligned}
& \left.E^{e}\right|_{\text {top }}=-\left(\frac{\sqrt{d_{12}{ }^{2}+2 \beta_{12}\left(\left.d_{12} E^{e}\right|_{\text {bottom }}+\left.0.5 \beta_{12} E^{e}\right|_{\text {bottom }} ^{2}\right)}+d_{12}}{\beta_{12}}\right) \\
& \left.E^{e}\right|_{\text {top }}=\frac{\sqrt{d_{12}^{2}+2 \beta_{12}\left(\left.d_{12} E^{e}\right|_{\text {bottom }}+\left.0.5 \beta_{12} E^{e}\right|_{\text {bottom }} ^{2}\right)}-d_{12}}{\beta_{12}}
\end{aligned}
$$

Following Reissner's formulation for large deformations of slender beams, the deformation of the beam actuated by mechanical and non-mechanical effect is studied. The beam is initially straight and subjected to the following internal shear force $Q$, normal force $N$ and bending moment $M$. Consider a differential element of the straight beam with length $d s$ at location $s$ before deformation as shown in figure 3a. After deformations, the current location of the element is $x(s)=\mathrm{s}+u(s)$ and $y(s)=v(s)$, where $u(s)$ and $v(s)$ are the displacement in the $x$ and $y$ axes, respectively. The kinematic relations for the displacements and rotation of the beam element are written as: 


$$
\begin{aligned}
& 1+u^{\prime}=(1+\varepsilon) \cos \phi \\
& v^{\prime}=(1+\varepsilon) \sin \phi
\end{aligned}
$$

where $\phi$ is the rotational angle of the cross-section when the beam deforms; thus, $\phi^{\prime}$ is the curvature of the deformed beam, $\varepsilon$ is the axial strain along the centroidal axis of the beam which, in case of no shear strain, is the same as the relative change of length of a differential element of the beam axis due to deformation, and for inextensible case this value is zero. ()' stands for the derivative with respect to $s$, which is the distance along the length of the beam measured from its left end. In this study, it is assumed that the beam is inextensible; in other words, the length of center line of the beam remains constant under deformation of the beam. Referring to figure $3 \mathrm{~b}$, and the moment equilibrium of the differential element lead to the following system of equations:

$$
M^{\prime}+(1+\varepsilon) Q=0
$$

A linear elastic constitutive relation for the beam (host structure) and equilibrium equations in terms of the axial force and bending moment lead to:

$$
M=E_{b} I \phi^{\prime}
$$

where $E_{b} I$ is the axial stiffness and bending stiffness of the beam, respectively.

Under a pure bending condition, deformation of a cantilever beam, Eqs. (13-15), can be solved analytically in order to obtain displacement components of the beam. In cases other than pure bending, for instance when mechanical loads are applied to the beam, closed form expressions for the deformed configuration are not necessarily available and a numerical method is then considered. One of the available numerical methods is nonlinear shooting method presented by Banerjee et al. [16] for obtaining solutions to large deformations of beams. The method is briefly described here and used for the analyses in Section 4. 
Consider a beam under a bending moment and concentrated forces $F_{x}$ and $F_{y}$, shown in figure 4 . The bending moment at a point $(x, y)$ of the cantilever beam is expressed as

$$
M(x, y)=F_{x}\left(d_{y}-y\right)-F_{y}\left(d_{x}-x\right)+M_{0}\left[u\left(s-l_{1}\right)-u\left(s-l_{2}\right)\right]
$$

where $u(s)$ is a unit step function, $F_{x}$ and $F_{y}$ are the components of the concentrated force in $x$ and $y$ directions, respectively, $d_{x}$ and $d_{y}$ are the $x$-direction and $y$-direction distance of the force from the clamped end, and $M_{\mathrm{o}}$ is the bending moment due to electric field input. By substituting Eq. (13) into moment-curvature relation in Eq. (15) and differentiating with respect to $s$, the first derivative of the curvature is given as:

$$
\phi^{\prime \prime}=\frac{1}{E_{b} I}\left(F_{y} \cos \phi-F_{x} \sin \phi\right)+M_{o}\left[\delta\left(s-l_{1}\right)-\delta\left(s-l_{2}\right)\right]
$$

where $\delta(s)$ is Dirac-Delta function. The boundary conditions for a cantilever beam are

$$
\begin{aligned}
& \left.\phi\right|_{s=0}=0, \\
& \left.\phi^{\prime}\right|_{s=L}=0
\end{aligned}
$$

The boundary value problem is transformed to an initial value problem (IVP) by estimating a value $m$ for curvature at $s=0$. So the initial values for Eq. (17) are

$$
\begin{aligned}
& \left.\phi\right|_{s=0}=0, \\
& \left.\phi^{\prime}\right|_{s=0}=m
\end{aligned}
$$

This initial value problem is then solved by $4^{\text {th }}$ order Runge-Kutta; then, the curvature at $s=L$ named $\lambda$, which is equal zero here, is used as a reference to evaluate the trial value $m$ that is expected to be close to the actual value for $\left.\phi^{\prime}\right|_{s=0}$. The trial value $m$ is then modified and this procedure is repeated for several steps until the calculated $\left.\phi^{\prime}\right|_{s=L}$ is close enough to $\lambda$ with a rate depending on the initial estimation for $m$. At each step, $k$, the formula for deriving $m_{k}$ is expressed as 


$$
m_{k}=m_{k-1}-\frac{\left.\phi^{\prime}\right|_{s=L}-\lambda}{\frac{\partial}{\partial m}\left(\left.\phi^{\prime}\right|_{s=L}\right)}
$$

It is seen that $\frac{\partial}{\partial m}\left(\left.\phi^{\prime}\right|_{s=L}\right)$ should be calculated as well. The right side of Eq. (17) depends on arc length, rotational angle, and curvature $f\left(s, \phi, \phi^{\prime}\right)$, and a new variable $\psi=\frac{\partial \phi}{\partial m}$ is defined. By introducing new variables as $\xi=\phi^{\prime}$ and $\zeta=\psi^{\prime}$, and after some algebraic manipulations, a system of first order differential equations is derived. In each step $k, 4^{\text {th }}$ order Runge-Kutta method is used to solve the IVP in Eq. (17):

$$
\begin{gathered}
\text { D.E. }\left\{\begin{array}{l}
\phi^{\prime}=\xi \\
\xi^{\prime}=f(s, \phi, \xi) \\
\psi^{\prime}=\zeta \\
\zeta^{\prime}=f_{, \phi} \psi+f_{, \xi} \zeta
\end{array}\right. \\
\text { I.C. }\left\{\begin{array}{l}
\left.\phi\right|_{s=0}=0 \\
\left.\xi\right|_{s=0}=m_{k} \\
\psi_{s=0}=0 \\
\left.\zeta\right|_{s=0}=1
\end{array}\right.
\end{gathered}
$$

which gives $\left.\xi\right|_{s=L}=\left(\left.\phi^{\prime}\right|_{s=L}\right)$ and $\left.\zeta\right|_{s=L}\left(=\frac{\partial}{\partial m}\left(\left.\phi^{\prime}\right|_{s=L}\right)\right)$. For the next step, the modified $m_{k+1}$ is calculated from Eq. (20) and the above procedure is repeated until the solution converges or $\left.\xi\right|_{s=L} \rightarrow \lambda$. Finally, the curvature of the beam is determined and the deformed configuration of the beam can be obtained from the kinematic relations Eq. (13).

\section{3) Governing Equations for Active Viscoelastic Beams}


In this section, a time-dependent parameter is taken into account for analyzing deformations in active beams with viscoelastic host structures. A constitutive relation for the axial strain in a linear viscoelastic beam is described as follows

$$
\varepsilon_{b}(s, \eta, t)=\int_{0^{-}}^{t} J(t-\tau) \frac{d \sigma(s, \eta, \tau)}{d \tau} d \tau
$$

where $\sigma$ is the axial stress and $J(t)$ is the creep function of the beam. The equation for the bending moment is obtained by multiplying both sides of Eq. (22) by a distance $\eta$ measured from a mid-surface of the beam and integrating both sides over the cross-section area of the beam:

$$
\int_{A} \eta \varepsilon_{b}(s, \eta, t) d A=\int_{A} \eta \int_{0^{-}}^{t} J(t-\tau) \frac{d \sigma(s, \eta, \tau)}{d \tau} d \tau d A=\int_{0^{-}}^{t} J(t-\tau) \frac{d M_{0}(x, y, \tau)}{d \tau} d \tau
$$

$M_{0}$ is an induced moment in the beam as expressed in Eq. (4). By considering the timedependence material properties of the beam, the moment and strain relations is:

$$
M_{0}=-\int_{-\frac{t_{b}}{2}}^{\frac{t_{b}}{2}} \int_{0^{-}}^{t} G(t-\tau) \frac{d \varepsilon_{b}}{d \tau} d \tau \eta d \eta=-\frac{b t_{b}^{3}}{t_{b}+\frac{t_{p}}{2}} \int_{0^{-}}^{t} G(t-\tau) \frac{d \chi}{d \tau} d \tau
$$

where $\chi$ is strain measure defined here as $K \varepsilon_{p}$, where $K=\frac{t_{p}\left(t_{b}+t_{p}\right) E_{p}}{\frac{1}{6} t_{b}^{2} E_{b}+t_{p}\left(t_{b}+t_{p}\right) E_{p}}$ and $G(t)=E_{b}(t)$ is the relaxation function which is related to the creep function as follows

$$
1=J(0) G(t)+\int_{0^{+}}^{t} G(t-\tau) \frac{d J(\tau)}{d \tau} d \tau
$$


As stated above, the mechanical response of the actuators is assumed linear elastic. Thus, $\varepsilon_{p}$ is given in Eq. (8) or (9).

By assuming no longitudinal extension of the centroidal axis of the beam under pure bending moment, the strain can be written in terms of the curvature as $\varepsilon=-\eta \phi^{\prime}$. Thus, Eq. (23) is rewritten as

$$
I_{y} \phi^{\prime}=J(t) M_{0}(s, 0)+\int_{0^{+}}^{t} J(t-\tau) \frac{d M_{0}(s, \tau)}{d \tau} d \tau
$$

By solving Eq. (26) for curvature, the deformed configuration of the beam can be obtained in a similar way as for the elastic case.

\section{4) Boundary Value Problems}

This section presents several boundary value problems for elastic and viscoelastic beams having several arrangements of piezoelectric patches. Linear and nonlinear electro-mechanical responses are considered for the piezoelectric patches. Analyses of shape changing in smart beams controlled by electric field inputs are presented.

\section{1) Elastic beam with pairs of piezoelectric patches}

Bending of smart cantilever beams with several pairs of piezoelectric patches is presented. Figure 5a shows an undeformed cantilever beam with a pair of piezoelectric patches with length $l_{p}$ attached to the top and bottom surface of the beam. The piezoelectric patches are assumed to be homogeneous having the same width as the beam.

From Eqs. (13) and (15), the closed form solution for the displacement components, $u$ and $v$, in term of $s$ are: 


$$
\begin{aligned}
& \left\{\begin{array}{l}
u=0 \\
v=0
\end{array} \quad \begin{array}{l}
u=-\frac{E_{b} I}{M_{o}} \sin \frac{M_{0}\left(l_{1}-s\right)}{E_{b} I}-s+l_{1} \\
v=\frac{E_{b} I}{M_{o}}\left(\cos \frac{M_{0}\left(l_{1}-s\right)}{E_{b} I}-1\right) \quad l_{1}<s<l_{1}+l_{p}
\end{array}\right. \\
& \left\{\begin{array}{l}
u=\left(\cos \frac{M_{0} l_{p}}{E_{b} I}-1\right) s+l_{1}+\frac{E I}{M_{o}}\left(\sin \frac{M_{0} l_{p}}{E_{b} I}\right)-\left(l_{1}+l_{p}\right) \cos \frac{M_{0} l_{p}}{E_{b} I} \\
v=-\left(\sin \frac{M_{0}\left(l_{1}-s\right)}{E_{b} I}\right) s+\frac{E I}{M_{o}}\left(\cos \frac{M_{0} l_{p}}{E_{b} I}-1\right)+\left(l_{1}+l_{p}\right) \sin \frac{M_{0} l_{p}}{E_{b} I} \quad l_{1}+l_{p}<s
\end{array}\right.
\end{aligned}
$$

It is possible to attach several pairs of piezoelectric patches along the length of the beam and different magnitude of electric field can be applied to the piezoelectric patches in order to induce various shape changes. Consider now a cantilever beam with two piezoelectric patches and geometric quantities shown in figure $5 \mathrm{~b}$. The moments generated by the two pairs of the piezoelectric patches from left to right are $M_{1}$ and $M_{2}$, respectively. The closed form solutions for the displacement components of the elastic beam are:

$$
\begin{aligned}
& \left\{\begin{array}{l}
u=0 \\
v=0
\end{array}\right. \\
& \left\{\begin{array}{l}
u=-\frac{E_{b} I}{M_{o}} \sin \frac{M_{1}\left(l_{1}-s\right)}{E_{b} I}-s+l_{1} \\
v=\frac{E_{b} I}{M_{o}}\left(\cos \frac{M_{1}\left(l_{1}-s\right)}{E_{b} I}-1\right) \quad l_{1}<s<l_{1}+l_{p 1}
\end{array}\right. \\
& \left\{\begin{array}{l}
u=\left(\cos \frac{M_{1} l_{p 1}}{E_{b} I}-1\right) s+l_{1}+\frac{E_{b} I}{M_{o}}\left(\sin \frac{M_{1} l_{p 1}}{E_{b} I}\right)-\left(l_{1}+l_{p 1}\right) \cos \frac{M_{1} l_{p 1}}{E_{b} I} \\
v=-\left(\sin \frac{M_{1}\left(l_{1}-s\right)}{E_{b} I}\right) s+\frac{E_{b} I}{M_{o}}\left(\cos \frac{M_{1} l_{p 1}}{E_{b} I}-1\right)+\left(l_{1}+l_{p 1}\right) \sin \frac{M_{1} l_{p 1}}{E_{b} I} \quad l_{1}+l_{p 1}<s<l_{2}
\end{array}\right.
\end{aligned}
$$

for $l_{2}<s<l_{2}+l_{p 2}$ : 


$$
\left\{\begin{array}{l}
u=-\left(\frac{E_{b} I}{M_{2}}\right) \sin (\varphi)-s+c_{4}^{(1)} \\
v=\left(\frac{E_{b} I}{M_{2}}\right) \cos (\varphi)+c_{4}^{(2)}
\end{array}\right.
$$

where

$$
\begin{aligned}
& \phi=-\frac{M_{2}}{E_{b} I} s+\frac{M_{2} l_{2}-M_{1} l_{p 1}}{E_{b} I} \\
& c_{4}^{(1)}=l_{1}+\left(l_{2}-l_{1}-l_{p 1}\right) \cos \frac{M_{1} l_{p 1}}{E_{b} I}+E I\left(\frac{1}{M_{1}}-\frac{1}{M_{2}}\right) \sin \left(\frac{M_{1} l_{p 1}}{E_{b} I}\right) \\
& c_{4}^{(2)}=-\left(l_{2}-l_{1}-l_{p 1}\right) \sin \frac{M_{1} l_{p 1}}{E_{b} I}-\frac{E_{b} I}{M_{2}} \cos \left(\frac{M_{1} l_{p 1}}{E_{b} I}\right)-\frac{E_{b} I}{M_{1}}\left(1-\cos \frac{M_{1} l_{p 1}}{E_{b} I}\right)
\end{aligned}
$$

for $l_{2}+l_{p 2}<s$ :

$$
\left\{\begin{array}{l}
u=(\cos \phi-1) s+c_{5}^{(1)} \\
v=-(\sin \phi) s+c_{5}^{(2)}
\end{array}\right.
$$

where

$$
\begin{aligned}
\phi= & \frac{M_{1} l_{p 1}+M_{2} l_{p 2}}{E_{b} I} \\
c_{5}^{(1)}= & l_{1}+\left(l_{2}-l_{1}-l_{p 1}\right) \cos \frac{M_{1} l_{p 1}}{E_{b} I}-\left(l_{3}+l_{p 2}\right) \cos \left(\frac{M_{1} l_{p 1}+M_{2} l_{p 2}}{E_{b} I}\right) \\
+ & \frac{E_{b} I}{M_{2}}\left[\sin \left(\frac{M_{1} l_{p 1}+M_{2} l_{p 2}}{E_{b} I}\right)-\sin \frac{M_{1} l_{p 1}}{E_{b} I}\right]
\end{aligned}
$$

$$
\begin{aligned}
c_{5}^{(2)}= & \left(l_{1}+l_{p 1}-l_{2}\right) \sin \frac{M_{1} l_{p 1}}{E_{b} I}+\left(l_{3}+l_{p 2}\right) \sin \left(\frac{M_{1} l_{p 1}+M_{2} l_{p 2}}{E_{b} I}\right)+\frac{E_{b} I}{M_{2}}\left[\cos \left(\frac{M_{1} l_{p 1}+M_{2} l_{p 2}}{E_{b} I}\right)-\cos \frac{M_{1} l_{p 1}}{E_{b} I}\right] \\
& -\frac{E_{b} I}{M_{1}}\left(1-\cos \frac{M_{1} l_{p 1}}{E_{b} I}\right)
\end{aligned}
$$


In general, any number of piezoelectric patches can be added to the beam and a closed form solution for deformation of the beam under induced bending moments can be derived. The general procedure is to calculate the rotational angle of the axis of the deformed beam $\phi$ from moment-curvature constitutive relation at first and then deriving deformation components in every location of the beam from the kinematics relation. In calculating the displacements, the compatibility condition (continuity displacements) at the interface of each two adjacent segments of the beam is considered as the boundary conditions for analyzing the deformation of each segment. Consider a part of the beam with multiple piezoelectric patches on its top and bottom as shown in figure 6. Continuity of the displacement between the adjacent segments of the beams leads to boundary conditions as follows:

$$
\begin{gathered}
\phi_{i}\left(s_{i}\right)=\phi_{i}\left(s_{i}\right) \\
\left.u_{i}(s)\right|_{s=s_{i}}=\left.u_{i+1}(s)\right|_{s=s_{i}} \\
\left.v_{i}(s)\right|_{s=s_{i}}=\left.v_{i+1}(s)\right|_{s=s_{i}}
\end{gathered}
$$

From Eq. (13), the displacement components for segment $i$ and $i+1$, respectively, are:

$$
\begin{aligned}
& u_{i}(s)=\int \cos \phi_{i}(s) d s-s+c_{i}^{(1)} \\
& v_{i}(s)=\int \sin \phi_{i}(s) d s+c_{(i)}^{(2)} \\
& u_{i+1}(s)=\sin \phi_{i+1}-s+c_{(i+1)}^{(1)} \\
& v_{i+1}(s)=-\cos \phi_{i+1}+c_{(i+1)}^{(2)}
\end{aligned}
$$

The constants $c_{i}^{(1)}, c_{i}^{(2)}, c_{i+1}{ }^{(1)}$ and $c_{i+1}{ }^{(2)}$ are derived from Eq. (29). It should be noted that the rotation of segment $i+1, \phi_{i+1}$, is constant due to no internal force or moment in the segment.

As mentioned earlier, the induced bending moment depends on the axial strain in the excited actuator patches which may be linearly or nonlinearly proportional to the applied electric field. The effect of linear and nonlinear response of the piezoelectric material on the deformation 
of a cantilever beam with two pairs of piezoelectric patches can be seen by comparing configuration of the actuated beam in figure 7. The materials properties of the components are considered as $E_{b}=4.9 \mathrm{GPa}, E_{p}=38.6 \mathrm{GPa}, d_{12}=0.18 \mathrm{~nm} / \mathrm{V}, \beta_{12}=8(\mathrm{pm} / \mathrm{V})^{2}$ where the linear and nonlinear piezoelectric coefficients are those reported for PZT G-1195 [17]. The segments of the beam with patches are drawn with thicker lines. As seen, the curvature of the deformed beam in segments without patch is zero and so those segments remain straight while in segments bonded with actuator patches, changes of curvature occur along the length of the segments and so the displacement components are nonlinear functions of $s$. As seen in the figure, assuming a linear response of piezoelectric material with respect to electric field underestimates the bending moment. The amount of maxium axial strains for the linear and nonlinear cases are $5.7 \times 10^{-4} \%$ and $3.8 \%$. In this example, the position and length of the patches are $l_{1}=0.25 L l_{2}=0.4 L$ and $l_{p 2}=l_{p 1}=0.1 L$, respectively and $E^{e}=0.3 \mathrm{MV} / \mathrm{m}$.

\section{2) Elastic beam with multiple piezoelectric patches and end force}

The deformation of a cantilever beam actuated electrically by the piezoelectric patches and mechanically by a concentrated force at its free end is analyzed by solving Eq. (17) using the nonlinear shooting method. Three smart beams are studied and the responses are shown in figure 8. The analyses are done by considering $-F_{x} L^{2} / E_{b} I=F_{y} L^{2} / E_{b} I=0.2$ where $F_{x}$ and $F_{y}$ are components of the applied $F$; all the patches are assumed to be the same length and thickness and subjected to the same amount of electric field, $E^{e}=10 \mathrm{KV} / \mathrm{m}$ such that for each pair $M_{0} L / E_{b} I=$ 1. 


\section{3) Shape control of the actuated elastic beams}

Another example is folding of slender beams under actuation of multiple patches, which may be used in deployable systems. Figure 9 shows a beam with five pairs of patches attached to them. The beam is originally straight (figure 9a) with patch labels shown. Nonlinear response in terms of electric field is assumed for the actuators and electric field $E^{e}=0.42 \mathrm{MV} / \mathrm{m}$ is applied to the patch number one and it generates bending moment as $M_{0} L / E_{b} I=31$ which folds the tip of the beam into a ring- shape configuration (figure $9 \mathrm{~b}$ ). Then, the next patch undergoes the same magnitude of electric field and another pair of moment induced to the beam so larger part of the beam folds (9c). This process of actuating can be continued by patches three, four and five, and the ring- shape moves to the left as seen in figure 9c-e respectively. It should be noted that since the beam and the patches are elastic, the configuration of the beam does not depend on the history of applied electric fields, as in the case of viscoelastic beams which is discussed later.

\section{4) Viscoelastic composite beams with piezoelectric patches}

A viscoelastic free beam with two pair of patches bonded to two sides of the beam symmetrically is considered. The patches with length $l_{p 1}=l_{p 2}=0.1 \mathrm{~L}$ are attached at left and right ends of the beams, respectively. The nonlinear strain of the piezoelectric material is considered again as quadratic form according to Eq. (9). The properties of the elastic patches are the same as before but for the beam time dependent relaxation function is considered as $G_{b}(t)=1\left(1-0.95\left(1-e^{-t / 2}\right)\right)$ GPa. Its corresponding creep function is $J(t)=10^{-8}\left(2-1.9 e^{-t / 40}\right) \mathrm{Pa}^{-1}$ according to Eq. (25). The electric field stimulus is chosen in amount of $0.1 \mathrm{MV} / \mathrm{m}$. The time-dependent deformation of the beam at different times is obtained and shown in figures 10. It is seen that the curvature of the 
beam at positions covered with actuator layers increases gradually over time, which is due to the creep deformation of the viscoelastic beam and relaxing the bending rigidity of the beam with time.

Finally, with the same properties and electric stimulus, the cantilever beam shown in figure $9 \mathrm{a}$ is analyzed. According to figure $9 \mathrm{a}$, only an excitation of patches number one and two are considered this time. Figures 11 shows the configurations of the active beam at different times under actuation of the two pair of actuators. Similar to the case seen in figure 10, as time increases, the beam gradually is folded and at some point it almost turns into the shape seen in figure $9 \mathrm{c}$. The maximum axial strain in the beam is obtained as $6.4 \%$ at $\mathrm{T}=5 \mathrm{sec}$.

\section{4) Conclusion}

Large deformations of smart flexible cantilever beams due to electric fields applied to piezoelectric patches have been studied. The flexible beams having linear elastic and viscoelastic responses have been considered. Pairs of piezoelectric actuators are placed on top and bottom of the beams so that when the electric fields are prescribed on them, the smart beams are under bending. Reissner's large deformation beam theory is adopted for the analysis. The expression for the induced moment by the piezoelectric patches is presented by considering both linear and nonlinear electro-mechanical response of the piezoelectric material. Finally, through several examples, different configurations of flexible actuated elastic and viscoelastic beams are studied, which can be used for preliminary design of active flexible structures. As seen in the analyses when the magnitude of electric field inputs is sufficiently large, linear and nonlinear electromechanical responses of the piezoelectric patches lead to significantly different deformations. 
When viscoelastic beams are considered, time plays an important role for deformed configuration of the beam due to the relaxation behaviors of the viscoelastic beams.

\section{Acknowledgement}

This research is sponsored by the Air Force Office of Scientific Research (AFOSR) under grant FA9550-14-1-0234 and the National Science Foundation (NSF) under grant CMMI-1437086.

\section{REFERENCES}

1. B.-T. Wang, C.A. Rogers, Modeling of finite-length spatially distributed induced strain actuators for laminated beams and plates, in Journal of Intelligent Materials and Systems, 1991. p. 38-58.

2. P. Gaudenzi, D. Giarda., F. Morganti, Active microvibration control of an optical payload installed on the Artemis spacecraft, in 8th international conference on adaptive structures and technologies, ICAST 97, 1997: Wakayama. p. 265-275.

3. D.G. Fertis, Nonlinear structural engineering. 2006: Springer.

4. C. kimball, L.-W. Tsai, Modeling of flexible beams subjected to arbitrary end loads. ASME Journal of Mechanical Design, 2002. 124: p. 223-234

5. L. L. Howell, A. Midha, Parametric deflection approximations for end-loaded large deflection beams in compliant mechanisms. ASME Journal of Mechanical Design, 1995. 117: p. 156-165.

6. Y. Tada, G. Lee, Finite element solution of elastic problem of beams. International Journal of Numerical Methods and Engineering, 1970. 2: p. 229-241.

7. T. Y. Yang, Matrix displacement solution of elastica problems of beams. International Journal of Solids and Structures, 1973. 9: p. 829-842.

8. A. Chajes, J. Churchill Nonlinear frame analysis by finite element methods. Journal of Structural Engineering, 1987. 113: p. 1221-1235.

9. E. Reissner, On one-dimensional finite strain beam theory: the plane problem. Journal of Applied Mathematics and Physics, 1972. 23: p. 795- 804.

10. H. Irschik, J. Gerstmayr, A continuum mechanics based derivation of Reissner's largedisplacement finite-strain beam theory: the case of plane deformations of originally straight BernoulliEuler beams. Acta Mechanica, 2009. 206: p. 1-21.

11. P. M. Naghdi, L. Vongsarnpigoon, A theory of shells with small strain accompanied by moderate rotation. Archive for Rational Mechanics and Analysis, 1983. 83: p. 245-283.

12. A. R. Srinivasa, On a class of Gibbs potential-based nonlinear elastic models with small strain. Acta Mechanica, 2015. 226: p. 571-583.

13. B. J. de Blonk, D.C. Lagoudas, Actuation of elastomeric rods with embedded two-way shape memory alloy actuators. Smart Materials and Structures, 1997. 7: p. 771-783.

14. I. G. Tadjbakhsh, D.C. Lagoudas, Actuation of elastomeric rods with embedded two-way shape memory alloy actuators. Smart Materials and Structures, 1993. 2: p. 71-81. 
15. S. G. Shu, D.C. Lagoudas, D. Hughes, J. T. Wen, Modeling of a flexible beam actuated by shape memory alloy wires. Smart Materials and Structures, 1997. 6: p. 265-277.

16. A. Banerjee, B. Bhattacharya, A. K. Mallik, Large deformation of cantilever beams with geometric non-linearity: Analytical and numerical approaches. International Journal of Non-Linear Mechanics, 2008. 43: p. 366-376.

17. H. F. Tiersten, Electroelastic equations for electroded thin plates subject to large driving voltages Journal of Applied Physics 1993. 74(5): p. 3389 - 3393.

18. A. Muliana, C.-H. Lin, A multi-scale formulation for predicting non-linear thermo-electromechanical response in heterogeneous bodies. Journal of Intelligent Material Systems and Structures, 2011. 22: p. 723-738.

19. P. Tan, L. Tong, Micro-electromechanics models for piezoelectric-fiber-reinforced composite materials. Composites Science and Technology, 2001. 61(5): p. 759-769.

20. V. Tajeddini, C.-H. Lin, A. Muliana, M. Lévesque, Average electro-mechanical properties and responses of active composites. Computational Materials Science, 2014. 82: p. 405-414.

21. Sh. Ya-Peng, P. Ya-Fei, Stress and fracture analyses of viscoelastic large strain plane problem by finite element method, in Proceeding of the international conference on nonlinear mechanics, 1985: Shanghai. p. 1301-1306.

22. J. T. Holden, On the finite deflection of thin viscoelastic beams. International Journal of Numerical methods in engineering, 1972. 5: p. 271-275.

23. V.A. Baranenko, Large displacements of viscoelastic beams, Mechanics of Composite Materials, 1980. 15: p. 681-684.

24. K. Lee, Large deflection of viscoelastic fiber beams, Textile Research Journal, 2007. 77: p.47-51.

25. M.A. Vaz, M. Caire, On the large deflections of linear viscoelastic beams, International Journal of Non-linear Mechanics, 2010. 45: 75-81.

26. C. E. Beldica, H. H. Hilton, Nonlinear viscoelastic beam bending with piezoelectric controlAnalytical and experimental simulations. Composite Structures, 2001. 51: p. 195-203.

27. A. Muliana, Large deformation of nonlinear viscoelastic and multi-responsive beams. International Journal of Non-linear Mechanics, 2015. 71: 152-164.

28. E. F. Crawley, J. de Luis, Use of piezoelectric actuators as elements of intelligent structures. AIAA Journal, 1987. 25: p. 1373-1385. 


\section{Figures}

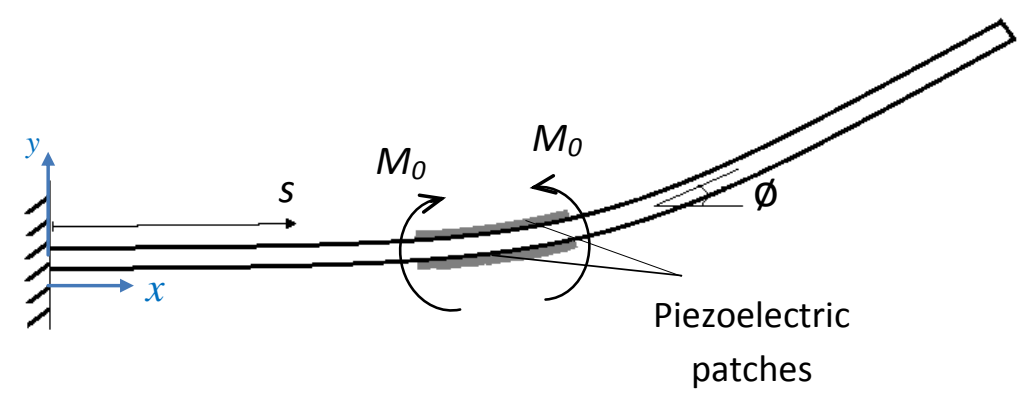

Figure 1. Deformation of a cantilever beam with a pair of piezoelectric patches to induced bending

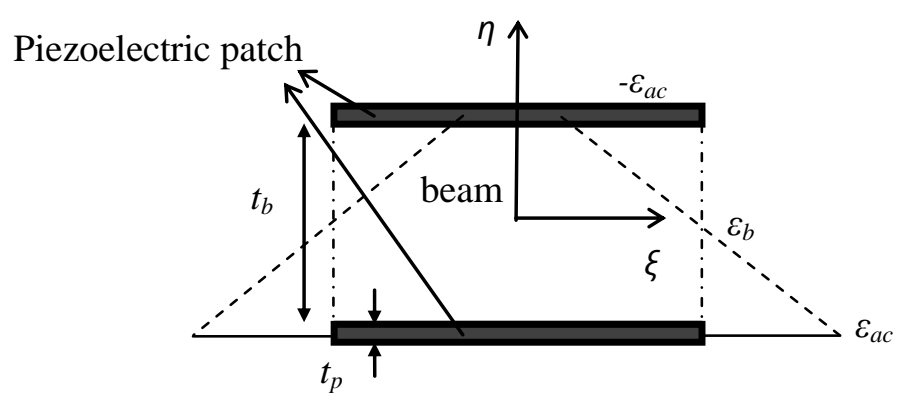

Figure 2. Strain distribution in the cross section of beam by an active piezoelectric patch 


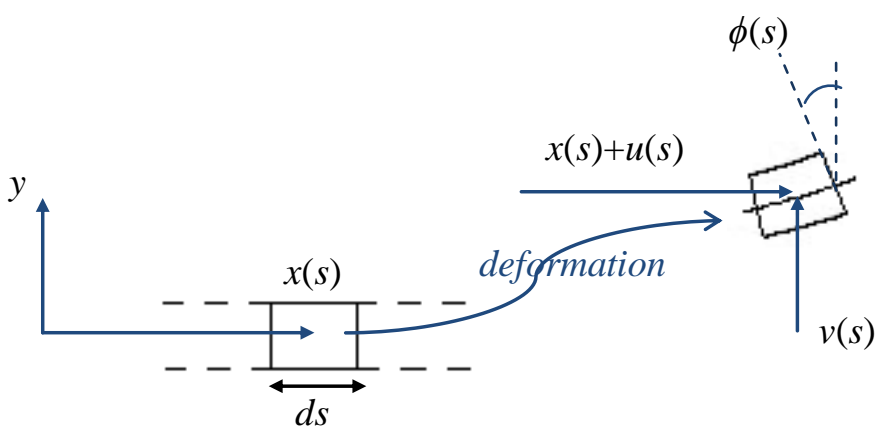

(a)

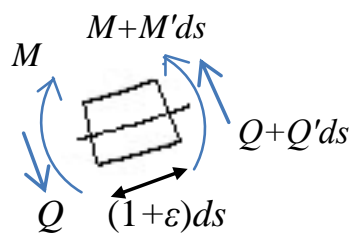

(b)

Figure 3. (a) Kinematic of an element of the beam before and after deformation and (b) free body diagram an element of the beam

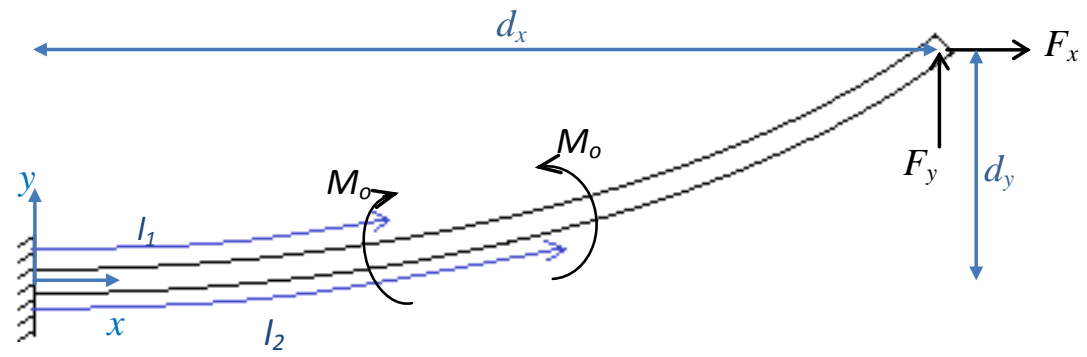

Figure 4. Configuration of cantilever beam under applied moment and force 


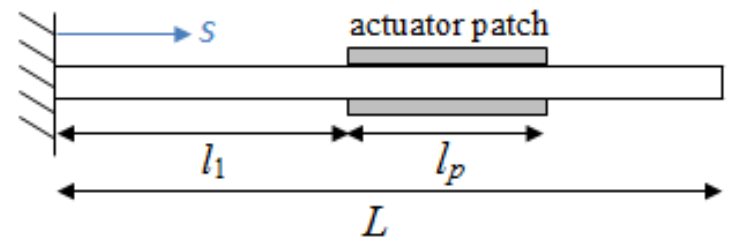

(a)

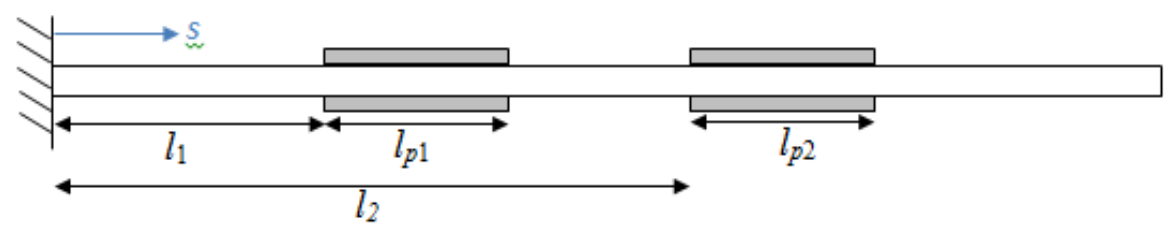

(b)

Figure 5. Catilever beam with one (a) and two (b) pairs of piezoelectric patches

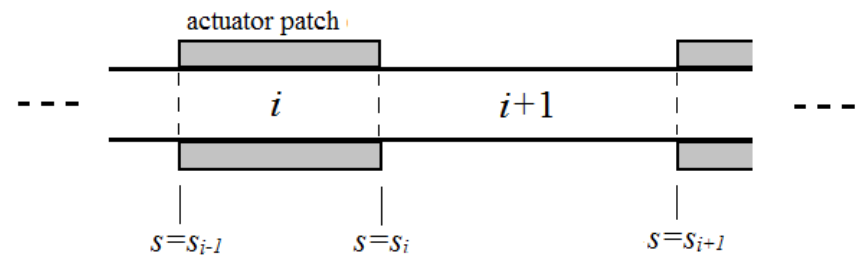

Figure 6. A part of the beam with multiple attached patches with pair $i$ shown 


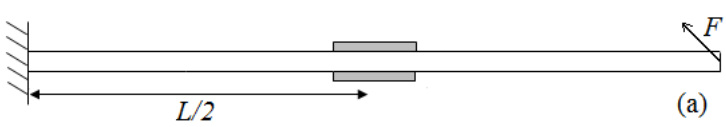

Figure 7. Deflection of the cantilever beam with three actuator patches made of piezoelectric material with (a) linear and (b) nonlinear behavior
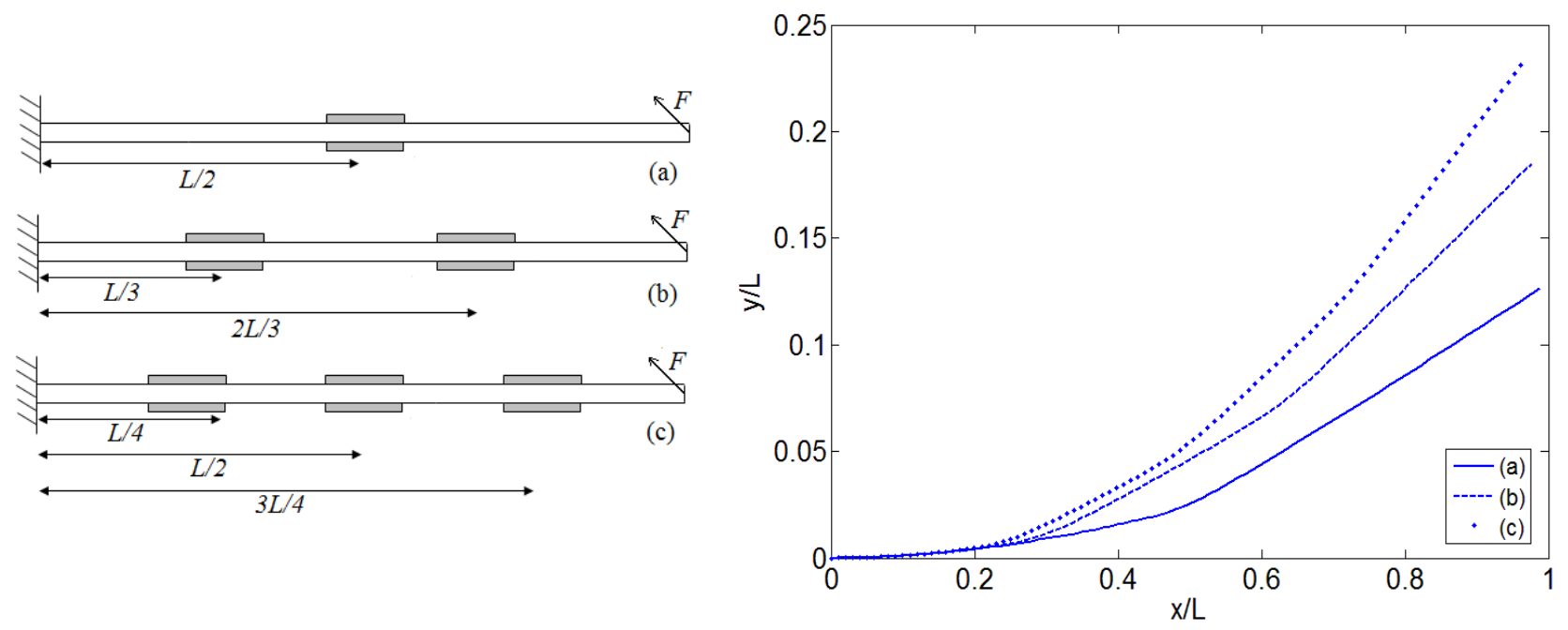

(a)

(b)

Figure 8. Deformation of cantilever beam with one (a), two (b) and three (c) pairs of patches under electro-mechancial actuation 
1

2

3

4

5

11

12

13

14

15

16

17

18

19

20

21

22

23

24

25

26

27

28

29

30

31

32

33

34

35

36

37

38

39

40

41

42

43

44

45

46

47

48

49

50

51

52

53

54

55

56

57

58

59

60

61

62

63

64

65

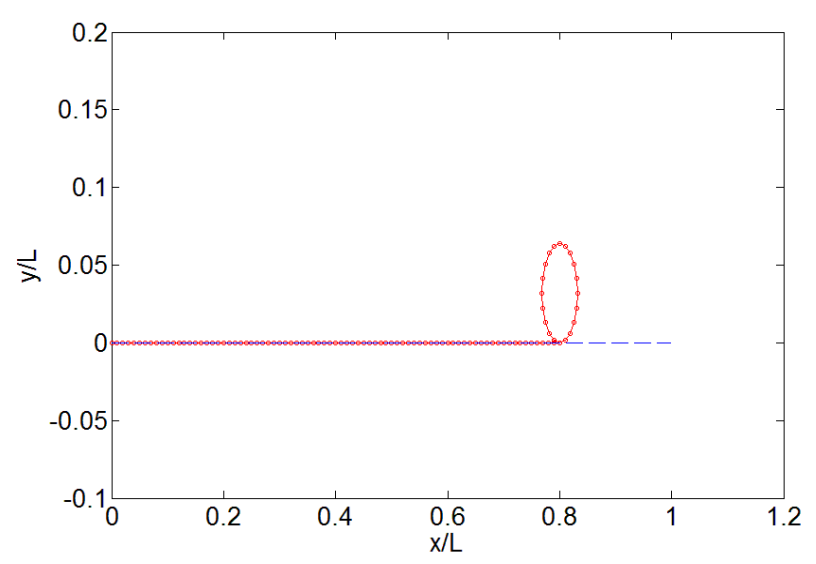

(b)

(a)
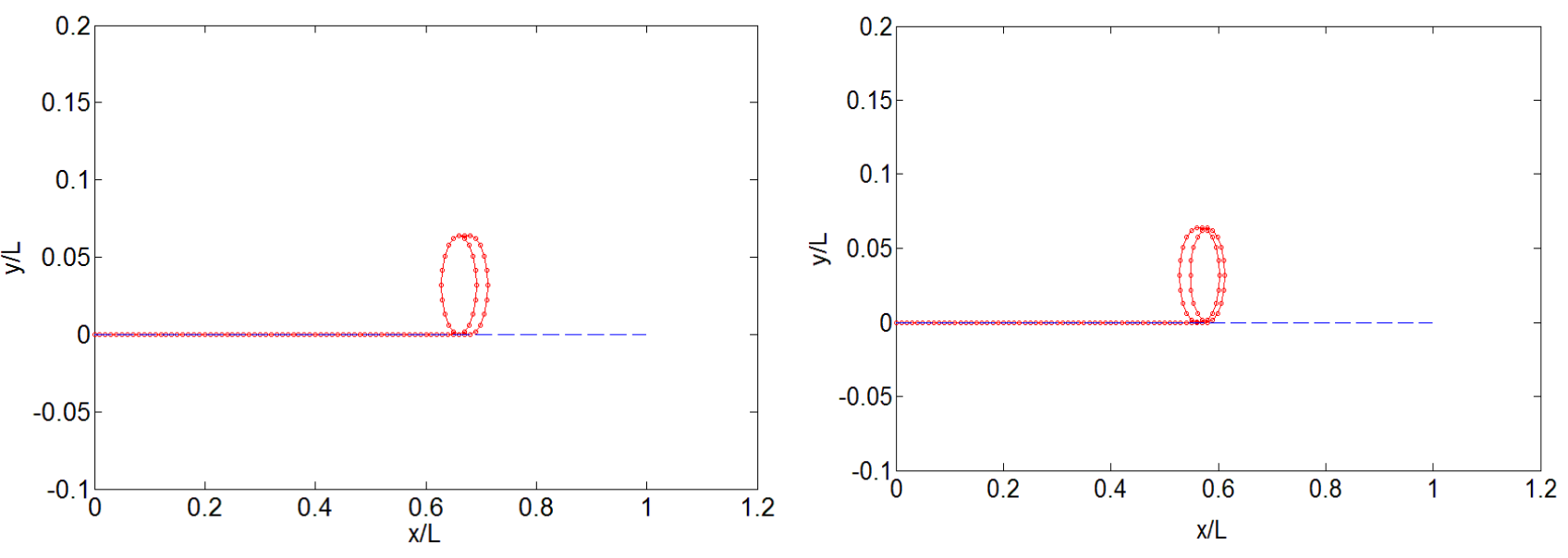

(c)

(d)

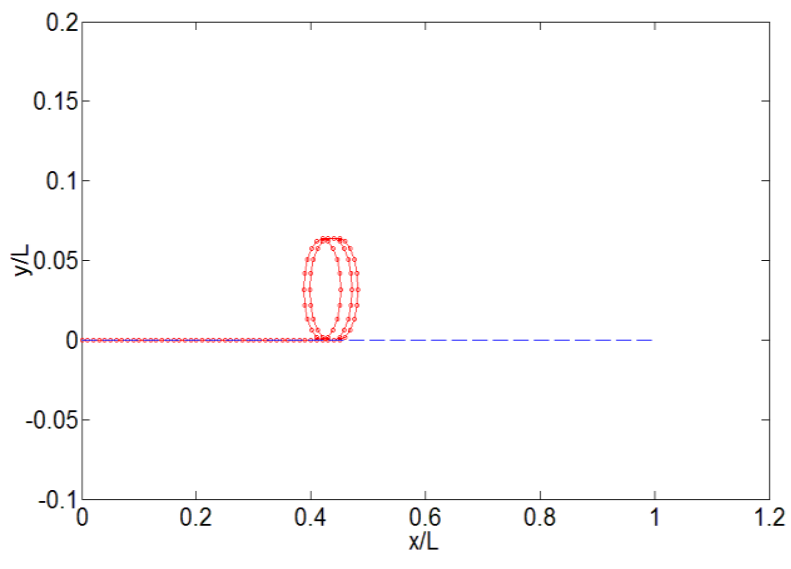

(e)

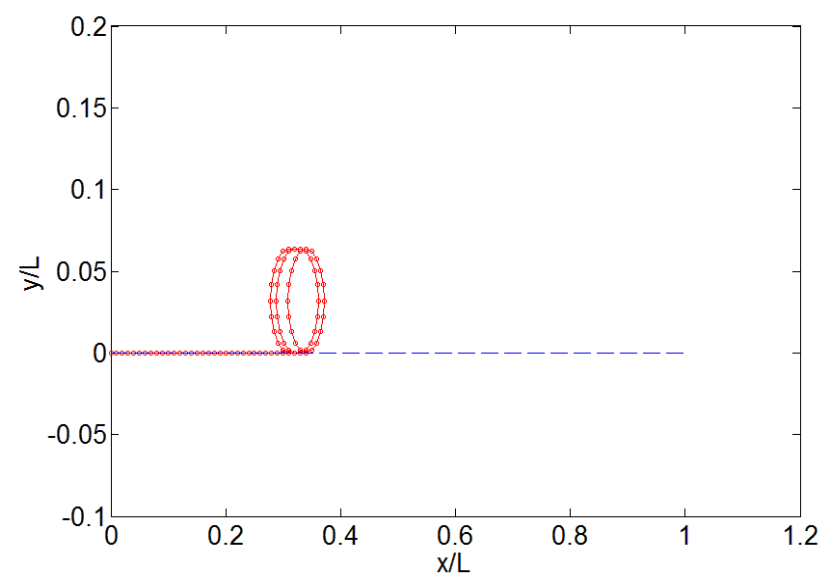

(f)

Figure 9. Rolling configuration of an originally straight cantilever beam (a) under actuation of one to five active pair of patches (b-f) 
1

2

3

4

5

6

10

11

12

13

14

15

16

17

18

19

20

21

22

23

24

25

26

27

28

29

30

31

32

33

34

35

36

37

38

39

40

41

42

43

44

45

46

47

48

49

50

51

52

53

54

55

56

57

58

59

60

61

62

63

64

65

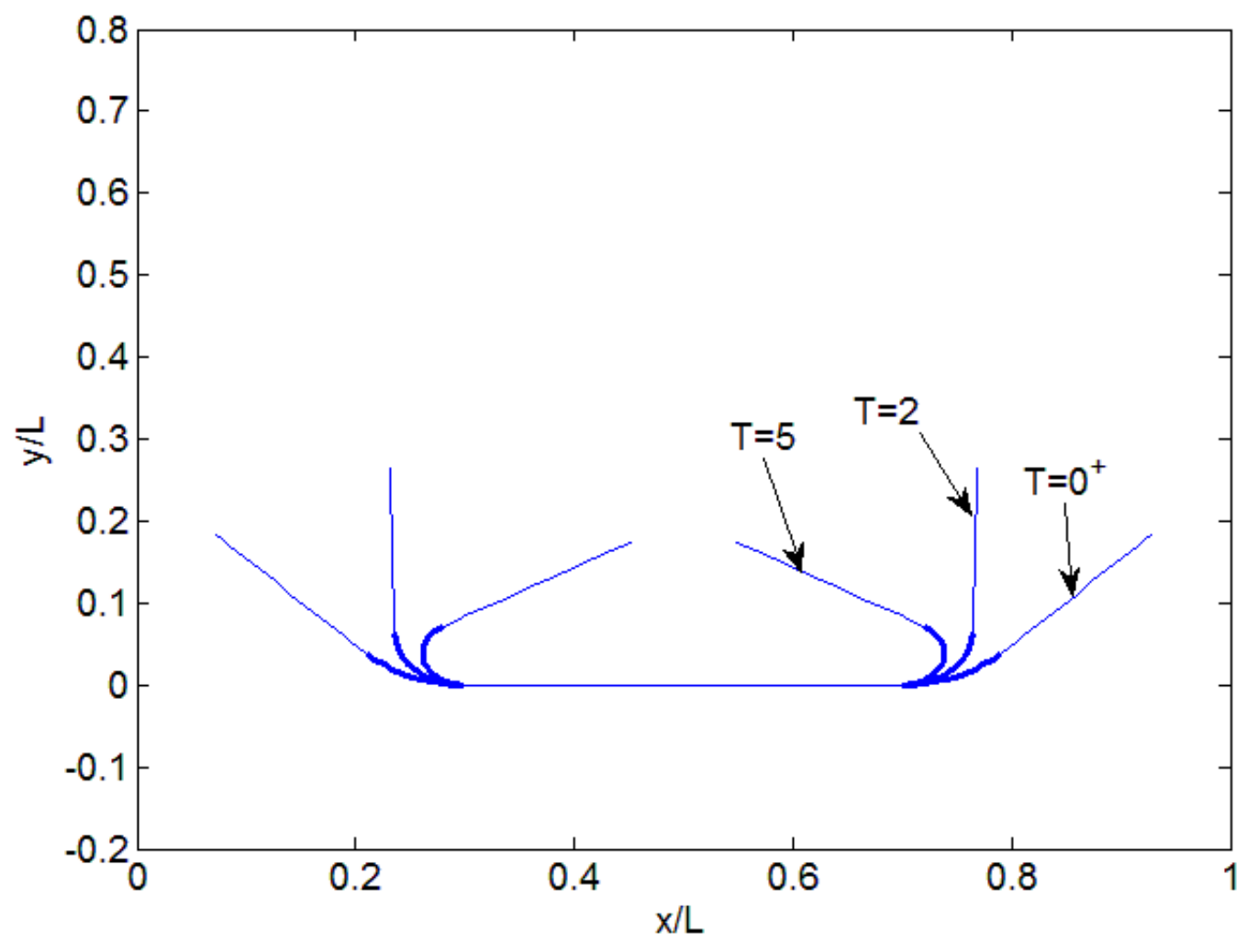

Figure 10. Deformation of a free viscoelastic beam with two pairs of active patch 
Figure 11. Configuration of an originally straight (at $T<0$ ) cantilever viscoelastic beam under actuation of two active pairs of patches 